\title{
CXLIII.-The Absorption Spectra of Aniline and its Homologues as Vapours, as Liquids, and in Solution.
}

\section{By John Edward Purvis.}

ThE aim of this communication is to give an account of a comparative study of the absorption spectra of aniline and its liquid homologues, in the state of vapours, liquids, and in solution, in order to ascertain, under these conditions, (1) the nature of the influence on the absorption of the radiant energy, and (2) how far the replacement of the hydrogen atoms by simple groups in the nucleus, or in the side-chain, affects the extent and type of the absorption bands.

The substances examined were aniline, mono- and di-methylaniline, mono- and di-ethylaniline, $o$ - and $m$-toluidine, $o$-3-xylidine, $m$-2-xylidine, mesidine, and benzylamine. The substances were distilled several times before use.

\section{Experimental.}

Vapours.

The absorption spectrum of the vapour of aniline in a tube 1-dem. long, and at $35^{\circ}$, has been studied by Pauer (Wied. Ann., $1897,61,374)$, who measured the positions of sixteen absorption bands, employing the radiant energy of the $\mathrm{Cd}$ spark. More recently, Grebe (Diss., Bonn, 1905; and Zeitsch. wiss. Photochem., $1905,3,363)$ studied the vapour of aniline at $25-30^{\circ}$, and used the continuous spectrum obtained when a powerful spark was passed between aluminium poles immersed in water. He noted the presence, and measured the positions of twenty-eight bands. Neither of these observers, however, investigated the effect of varying the temperature and pressure.

The author has already given an account of the absorption spectra of the vapours of pyridine and some of its derivatives (this 
Homologues as VApours, AS LIQUIDS, AND IN SOLUTION. 1547

vol., pp. 692 and 1035), and the apparatus used in this investigation is described in the first cited paper. Each band was separately measured at the different temperatures and pressures, and on the more refrangible edge.

\section{Aniline.}

Table $I$.

The absorption bands in aniline vapour at various temperatures and pressures in a column 2-dcm. long. Barometric pressure = $763 \mathrm{~mm}$.

Abbreviations: $\quad$ w. =weak; $\bmod$. str.=moderately strong; str. =strong; v.w. = very weak; r.w.= rather wide ; narr. =narrow ; mod. $\mathrm{w} .=$ moderately weak; $\mathrm{v}$. str. = very strong. The brackets of the wave-lengths represent the measurements of similar related bands; they are not measurements of the width of the bands.

$$
763 \mathrm{~mm} \text {. }
$$

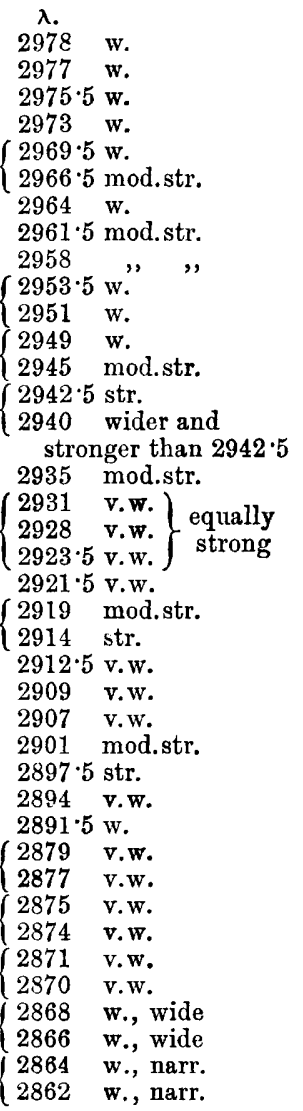

$30^{\circ}$.

$807 \mathrm{~mm}$.

$$
\lambda \text {. }
$$

2978 mod.w.

2977 w.

$2974 \cdot 5$ mod.str.

2973 mod.w.

$\{2970.5$ mod.str.

$\{2967$

2964

2962

2959

w.,, r.w.

mod.str.

$\{2954.5$ w.,, r.w.

2951.5 w., r.w.

$\{2950.5$ mod.str., narr.

2946

2943

.str.

2940 wider and

stronger than 2943

2934 mod.str.

$2930 \mathrm{w}$.

$2927 \cdot 5 \mathrm{w}$.

2924 w.

2921.5 w., narr.

(2918 str.

$\{2913.5$ wider and

stronger than 2918

$2912 \cdot 5$ v.w.

2910 w., wide

2907

2900

2897

2894

2891

w., narrower

than 2910

mod.str., narr.

str.

v.w.

mod.str., narr.

$\{2878.5 \mathrm{w} .$, narr.

$\{2877 \cdot 5$

\{ 2876

2875

2872

2871

2868

$\{2866$

,"
w., "wide

$45^{\circ}$.

$833 \mathrm{~mm}$.

$\lambda$.

2981 w., narr.

2978 mod.str., narr.

2977 w.

2975 str., narr.

2973 mod.str.

$2970 \cdot 5$ mod.str., wider than 2973

2966.5 mod.str.

2963

2962

2958.5, ,, narr.

$\{2954 \cdot 5$ w., wide

$\{2951$

\{ 2949

2946

mod.str., narr.

\{ $2942 \cdot 5$ str., "wide

2939

2934

( 2931 w., wide

$2928 \cdot 5$

$2924 \cdot 5$,",

$2921 \cdot 5$ w., narr.

( 2918 str., wide

\{2913.5 ,, ,

2912 v.w.

$2909 \cdot 5$ w., narr.

2906 w., wide

2900

2897 v.w., wide

2893 w.

2891 mod.str., wide

$\{2878 \cdot 5$ v.w.

$\{2877 \cdot 5 \mathrm{v} . \mathrm{w}$.

$\{2876$ v.w.

$\{2875$ v.w.

$\{2872$ v.w.

$\{2871$ v.w.

General absorption began about $\lambda 2850$. 36 bands. 
1548 PURVIS : THE ABSORPTION SPECTRA OF ANILINE AND ITS

$14^{\circ}$.

$763 \mathrm{~mm}$.

$\lambda$.

2859.5 str., wide

2857 mod.str.

$\{2856$ w., narr.

$2853.5 \mathrm{v} . \mathrm{w}$.

$\left\{\begin{array}{l}2851.5 \text { v.w., narr. } \\ 2848.5, " \text { equal- } 2857 \text { mod.str. }\end{array}\right\}$

2846

$\{2844$

2841

2838

$\{2836$

$2834.5 \mathrm{w}$.

$\{2832 \mathrm{w}$.

$\left\{\begin{array}{cc}2830 & \text { mod.w. } \\ 2828 & \text { " equally }\end{array}\right\}$

$\left\{\begin{array}{l}2828 \\ 2826 \cdot 5\end{array}\right.$

2821 head of wide band

(2814.5 v.w.

2810.5 v.w.

2807 v.w., stronger than other two

2804.5 mod. str.

$2798.5 \mathrm{w}$.

$\{2794 \cdot 5 \mathrm{w}$.

$\{2791 \cdot 5 \mathrm{w}$.

2787 w., wide

2782 w., wide

2781 v.w.

2779 v.w.

$(2770$ v.w.

$\{2768.5$ v.w.

2766 v.w.

$\{2764 \cdot 5$ v.w.

2759 v.w.

$2757 \cdot 5 \mathrm{v} . \mathrm{w}$.

2755 v.w.

0,739 v.w.

2737 v.w.

2729 w.

2730 mod.str.

2726 v.w.

2725 v.w.

2722 v.w.

$2720 \cdot 5$ v.w., wide

$2717 \cdot 5$, , ,

General absorption began at about $\lambda 2400$; $C d$ lines 2321, 2313, 2288, 2265, 2194, 2144 were just visible. $30^{\circ}$.

$807 \mathrm{~mm}$.

$\lambda$.

$\left\{\begin{array}{l}2864 \\ 2862\end{array}\right.$

w., narr.

w., stronger

than 2864

$2859 \cdot 5$ str., wide

2851.5 v.w., narr.

2849

2847

( 2844.5 v.w., f."wide

$2841.5,,$,

$\{2838$ w., narr.

$\{2836$

$2834 \cdot 5$

$\{2832 \cdot 5$

( 2830.5 r.w.

2828

$2820^{\circ}$

2821 head of strong wide band

$\begin{cases}2816 & \text { w., } \\ 2812 & \text { narr. } \\ 2808.5 & \text { ", }\end{cases}$

$2805^{\circ}$ mod.'str.

2799 w., r.w.

$\{2795$

$\{2791 \cdot 5$

2787

2783

2781

277

2778 v.w., narr.

$\{2770.5$ w., narr.

$\{2768 \cdot 5$

$\left\{\begin{array}{l}2766 \cdot 5 \\ 2765 \cdot 5\end{array}\right.$

$2759 \cdot 5$ mod.str., wide

$2757 \cdot 5$ w., narr.

2754 mod.str., narr.

2744 v.w., narr.

2743 w., wide

$2737 \cdot 5$

2729 mod.str.

2730 stronger than 2729

$2726 \cdot 5 \mathrm{w}$., narr.

2725

2723

2720.5 w., "wide

$2717 \cdot 5$

,"

General absorption began at about $\lambda 2430$. $60^{\circ}$.

$863 \mathrm{~mm}$.

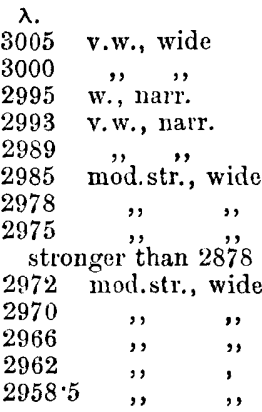

General absorption began at about $\lambda 2940$; the $\mathrm{Cd}$ line 2948 was just visible.

13 bands. $75^{\circ}$.

$893 \mathrm{~mm}$.

$\lambda$.

$3005 \mathrm{w}$, wide

3000 ,

2995 "

2993 ",

$2989 \quad$,

2985,

General absorption began at about $\lambda 2980$.

6 bands.

85 bands.

83 bands.

The absorption spectrum of aniline vapour was also observed at a constant temperature of $17.5^{\circ}$ and at gradually diminished pressures. The number and position of the bands were the same 


\section{HOMOLOGUES AS VAPOURS, AS LIQUIDS, AND IN SOLUTION. 1549}

as those observed at $14^{\circ}$ and $763 \mathrm{~mm}$. pressure in the above table; but they became sharper and weaker as the pressure decreased, and the position of general absorption was shifted towards the more refrangible regions of the spectrum. For example, under $754 \mathrm{~mm}$. pressure the general absorption began at about $\lambda 2400$, the $\mathrm{Cd}$ lines $2329,2321,2313,2288,2265,2194,2144$ were very weak; under $474 \mathrm{~mm}$. pressure the general absorption began at about $\lambda 2390$, and the Cd lines beyond were stronger; whilst under $24 \mathrm{~mm}$. pressure the general absorption began at about $\lambda 2370$, and the Cd lines were very strong.

The bands are more of the nature of strong lines than of what is usually understood by a band; and the width of many of them is measurable to the same degree and in the same sense as the images of many of the bright lines in emission spectra. They are not nearly so wide as an Angström unit, and the width of some of the narrow bands is not measurable at all. The constitution and appearance of the bands is completely different from those of the vapour of benzene as described by Hartley (Phil. Trans., 1907, A, 208, 475). Most of the latter appear to be wide bands, sharp and strong on the more refrangible edge.

The results prove that (1) an increase in the temperature and pressure produces a strengthening and widening of the bands, and the appearance of new bands on the less refrangible side; (2) a decrease in the pressure, the temperature remaining constant, produces a weakening and narrowing of the bands. At the same time, (3) an increase in the temperature and pressure produces a shift of the region of general absorption towards the red end; whilst (4) a decrease in the pressure, the temperature remaining constant, produces a shift in the opposite direction.

The bands observed in the vapour of aniline occur chiefly as a series of doublets and triplets; some of them are sharper on the more refrangible edge, whilst others have the edges equally sharp; some of the doublets appear to be equally strong, and some of them show the less refrangible of the two not quite so strong as the more refrangible one. Of the triplets, some have the three constituents equally strong, and of others the most refrangible constituent is the strongest. In addition to these general observations, an attempt has been made to arrange the bands in a definite and regular manner. The following table indicates fairly consistent differences in the wave-lengths of the majority of the bands, considered as a whole: 
AND ITS

$\begin{array}{rrrrr}3005 & & & & \\ 35 & 3000 & & & \\ 2970 & 33 & 2995 & & \\ 30 & 2967 & 31 & 2993 & \\ 2940 & 32 & 2964 & 31 & 2989 \\ 33 & 2935 & 33 & 2962 & 30 \\ 2907 & 34 & 2931 & 34 & 2959 \\ & 2901 & 34 & 2928 & 35 \\ 2870 & 33 & 2897 & 34 & 2924 \\ 21 & 2868 & 31 & 2894 & 33 \\ 2849 & 21 & 2866 & 30 & 2891 \\ & 2847 & 22 & 2864 & 29 \\ 2827 & 26 & 2844 & 23 & 2862 \\ 45 & 2821 & 29 & 2841 & 24 \\ 2782 & 40 & 2815 & 29 & 2838 \\ 43 & 2781 & & 2812 & 29 \\ 2739 & 44 & & 42 & 2809 \\ & 2737 & & 2770 & 40 \\ & & & 40 & 2769 \\ & & & 2730 & 43 \\ & & & & 2726\end{array}$

$\begin{array}{rrrrr}2985 & & & & \\ 31 & 2978 & & & \\ 2954 & 27 & 2977 & & \\ 33 & 2951 & 28 & 2975 & \\ 2921 & 32 & 2949 & 30 & 2973 \\ 42 & 2919 & 35 & 2945 & 30 \\ 2879 & 42 & 2914 & 33 & 2943 \\ 19 & 2977 & 39 & 2912 & 34 \\ 2860 & 20 & 2875 & 38 & 2909 \\ 24 & 2857 & 19 & 2874 & 38 \\ 2836 & 23 & 2856 & 20 & 2871 \\ 31 & 2834 & 24 & 2854 & 20 \\ 2805 & 35 & 2832 & 24 & 2851 \\ 39 & 2799 & 37 & 2830 & 23 \\ 2766 & 34 & 2795 & 38 & 2828 \\ 41 & 2765 & 36 & 2792 & \\ 2725 & 43 & 2759 & 34 & \\ & 2724 & 39 & 2758 & \\ & & 2720 & 41 & \end{array}$

The numbers run consecutively from left to right and diagonally, and the differences are differences in Ångström units between successive band intervals.

In addition to the regularity observed from comparing the whole of the bands, there are various minor groups which appear to be related. Of these, the following are a series of well-marked doublets whose most refrangible constituent is the strongest:
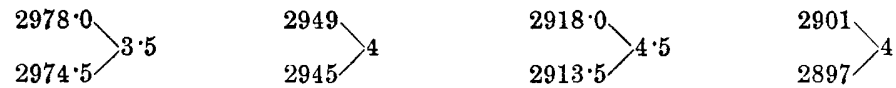

or, if the corresponding members of each group are arranged in tabular form, the following differences are observed:
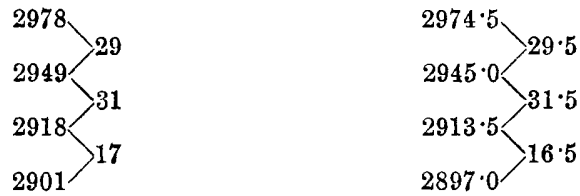

The following are a series of narrow doublets of similar appearance, the constituents of which are equally strong:

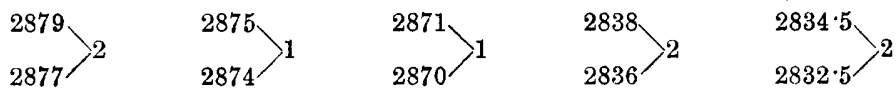

The bands 2830, 2828, 2826 are equally strong, and form a group by themselves with a constant difference of about 2 units; and the bands $2815,2811,2807$ form a group whose most refrangible constituent is the strongest, and there is a constant difference between them of about 4 units.

Photographs of the absorption spectra, in the 2-dcm. tube, of the vapour of the following substances were also taken under varying degrees of temperature and pressure. 
HOMOLOGUES AS VAPOURS, AS LIQUIDS, AND IN SOLUTION. 1551

Pressure

$t^{\circ}$. in $\mathrm{mm}$.

$14^{\circ} \quad 755 \quad$ The rays were transmitted to about $\lambda 2500$ and then absorbed; of the Cd lines, 2321, 2313, 2288, 2265, 2194, 2144 were just visible

$30 \quad 799$

$45 \quad 825$

60

75

90

100

$12 \cdot 5 \quad 765$

30

45

60

75

90

100

$13 \cdot 5 \quad 765$

$30 \quad 805$

45

60

75

90

100

12

766

The rays were transmitted to about $\lambda 2750$ and then absorbed; the Cd line 2573 was just visible.

The rays were transmitted to about $\lambda 3050$, and then all the rays beyond were absorbed.

855 The rays were transmitted to about $\lambda 2990$ and then absorbed.

885

915

931

The rays were absorbed from about $\lambda 3130$.

", ", $\quad$ ", $\quad \lambda 3150$.

\section{Dimethylaniline.}

The rays were absorbed from about $\lambda 2500$, but the strong $\mathrm{Cd}$ lines 2321 to 2144 were just visible.

The rays were absorbed from about $\lambda 2750$, but the Cd line 2573 was visible.

The rays were absorbed from about $\lambda 3020$, although the Cd line 2981 was visible.

The rays were absorbed from about $\lambda 3070$.

\begin{tabular}{|c|c|c|}
\hline & $"$ & " \\
\hline , & " & ," \\
\hline ", & " & " \\
\hline
\end{tabular}

\section{Monoethylaniline.}

The rays were absorbed from about $\lambda 2470$, but the strong $\mathrm{Cd}$ lines 2321 to 2144 were just visible.

The rays were absorbed from about $\lambda \mathbf{2 5 4 0}$. was well marked.

, $\quad \lambda 2700$, but the Cd line 2573

The rays were absorbed from about $\lambda 3050$.

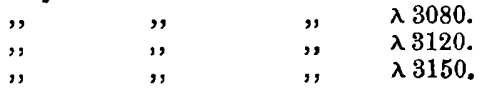

\section{Diethylaniline.}

The rays were transmitted to about $\lambda 2450$, and the $\mathrm{Cd}$ lines 2329 to 2144 were well marked.

The rays were absorbed from about $\lambda 3150$.

\section{Monomethylaniline.}

\section{o-Toluidine.}

$\begin{array}{rr}13 & 760 \\ 30 & 804 \\ 45 & 830 \\ 60 & 860 \\ 75 & 890 \\ 90 & 920 \\ 100 & 936\end{array}$

The rays were transmitted to about $\lambda 2470$; the Cd lines from 2329 to 2144 were well marked.

The rays were transmitted to about $\lambda 2540$; the Cd lines 2321, $2313,2288,2265,2194,2144$ were visible.

The rays were transmitted to about $\lambda 2700$; the $\mathrm{Cd}$ line 2573 was just visible.

The rays were transmitted to about $\lambda 2980$; the Cd line 2748 was just visible.

The rays were transmitted to about $\lambda 3060$.

$\begin{array}{llll}, ", & , & , & \lambda 3100 . \\ , & , & & \end{array}$




\section{m-Toluidine.}

$\begin{array}{cc} & \begin{array}{c}\text { Pressure } \\ t^{\circ}\end{array} \\ 13 & 759 \\ 45 & 829 \\ 45 & 889 \\ 75 & \\ 100 & 935\end{array}$

12

30

60

12

The rays were transmitted to about $\lambda 2480$; the Cd lines 2329 to 2144 were well marked.

The rays were transmitted to about $\lambda 2750$, the $\mathrm{Cd}$ line 2748 ending sharply ; the Cd line 2573 was just visible.

The rays were transmitted to about $\lambda 3050$; the Cd line 2980

was just visible.
The rays were transmitted to about $\lambda 3110$.

$$
\text { o-3-Xylidine (b. p. } 223^{\circ} \text { ). }
$$

752 The rays were transmitted to about $\lambda 2400$; the Cd lines 2329 to 2144 were well marked.

796 The rays were transmitted to about $\lambda 2450$; the $\mathrm{Cd}$ lines 2329 to 2144 were visible.

852 The rays were transmitted to about $\lambda 2600$; the $\mathrm{Cd}$ line 2573 was well marked.

$$
\text { m-2-Xylidine (b. p. } 215^{\circ} \text { ). }
$$

The rays were transmitted to about $\lambda 2250$; the $\mathrm{Cd}$ lines 2194 and 2144 were well marked.

The rays were transmitted to about $\lambda 2380$; the series of Cd lines from 2329 to 2144 were well marked.

The rays were transmitted to about $\lambda 2470$; the $\mathrm{Cd}$ lines from 2329 to 2144 were visible.

\section{Mesidine (b. p. $233^{\circ}$ ).}

759 The rays were transmitted to about $\lambda 2350$; the $\mathrm{Cd}$ lines 2321 to 2144 were strong.

829 The rays were transmitted to about $\lambda 2420$; the Cd lines 2321 to 2144 were well marked.

889 The rays were transmitted to about $\lambda 2550$; the Cd lines 2313 and 2268 were just visible.

935 The rays were transmitted to about $\lambda 3000$; the Cd lines 2880 , 2837 , and 2748 were visible.

\section{Benzylamine.}

12.5 752 The rays were transmitted to about $\lambda 2250$; the Cd lines 2194 and 2144 were well marked.

$30796 \quad$ The rays were transmitted to about $\lambda 2250$; the Cd lines 2194 and 2144 were visible.

45822 The rays were transmitted to about $\lambda 2420$; the $\mathrm{Cd}$ lines from 2329 to 2144 were well marked.

60852 The rays were transmitted to about $\lambda 2500$; the Cd lines from 2329 to 2144 were visible.

75882 The rays were transmitted to about $\lambda 2650$; the Cd line 2573 was well marked.

90910 The rays were transmitted to about $\lambda 2900$; the Cd lines 2880 2837 , and 2748 were visible. 
HOMOLOGUES AS VAPOURS, AS LIQUIDS, AND IN SOLUTION. 1553

\section{General Results of the Absorption Spectra of the Vapours.}

The results of the preceding observations of the absorption spectra of the vapours of aniline and its homologues prove that (1) aniline vapour has a considerable number of absorption bands which show relationships amongst themselves both in structure and in differences of wave-lengths; (2) under the influences of increased temperature and pressure the aniline bands become wider; weak bands become stronger; new bands make their appearance; and the general absorption is shifted towards the less refrangible region; (3) with decreased pressure, the temperature remaining constant, the aniline bands become sharper and weaker, and the region of general absorption is shifted towards the more refrangible region of the spectrum; (4) the most remarkable fact is that none of the homologues of aniline show any of the bands characteristic of aniline; (5) under the influences of increased temperature and pressure, the general absorption of the vapours of the homologues of aniline is shifted towards the less refrangible region.

\section{Solutions.}

The experimental methods employed in the study of the absorption spectra of solutions have been described before.

Aniline, Monomethylaniline, and Dimethylaniline.-The ultraviolet absorption spectra of alcoholic solutions of aniline have been described by Hartley and Huntington (Phil. Trans., 1879, 170, I, 257 ), who observed two bands at $\lambda 285-\lambda 274$ and $\lambda 240-\lambda 230$. Baly and Collie (Trans., 1905, 87, 1342) state that they repeated the observations with identically the same results. They also plotted the absorption curves of the bands in alcoholic solutions of monomethylaniline and dimethylaniline, and they show that the difference in the persistence of the band in the three cases is very marked. The absorption curve of dimethylaniline showed also a second band on the more refrangible side. The author found similar results to those of Baly and Collie in N/1000-solutions of these three substances, and the curves are therefore not reproduced.

Monoethylaniline and Diethylaniline.-The author has investigated the absorption spectra of the ultra-violet regions of $N / 1000$ alcoholic solutions of these substances. It will be noticed (Fig. 1) that diethylaniline shows a second band at about $1 / \lambda 3850$, which is absent in monoethylaniline. It is precisely similar to the phenomena observed by Baly and Collie in mono- and di-methylaniline. It is also noticeable that the characteristic band of these substances is not so persistent as the corresponding bands in aniline, mono- and di-methylaniline. 
1554 PURVIS : THE ABSORPTION SPECTRA OF ANILINE AND ITS

o-Toluidine.-Hartley (Trans., 1885, 47, 685) described a large, persistent band, the head of which was about $1 / \lambda 3040$.

m-Toluidine.-Baly and Ewbank (Trans., 1905, 87, 1358) described a large, persistent band, the head of which was about $1 / \lambda 3045$.

The author has repeated the observations with $N / 1000$-solutions of $o$-toluidine and $m$-toluidine, and found similar results. The curves therefore are not reproduced.

o-3-Xylidine and m-2-Xylidine.-N/1000-alcoholic solutions of

Fia. 1.

Oscillation frequencies.

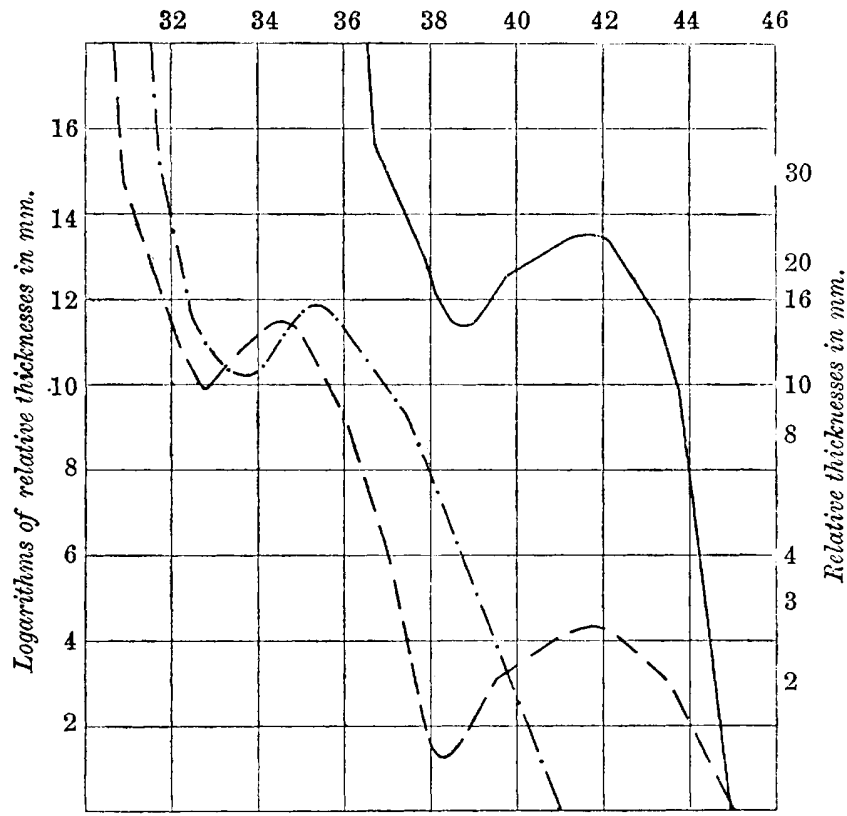

Continuous curve: N/100-alcoholic solution of benzylamine.

Dot and dash curve : N/1000-alcoholic solution of monoethylaniline.

Dash curve :

$\mathrm{N} / 1000$-alcoholic solution of diethylaniline.

these substances were observed, and their absorption curves have been drawn (Fig. 2). The general form of the curves is not unlike that of the toluidines. The bands are strongly persistent, and their positions are more towards the red end than the corresponding bands of the toluidines.

Mesidine.-An $N / 1000$-solution of this substance exhibits a band which is not very persistent (Fig. 2). It is of some interest to note 
HOMOLOGUES AS VAPOURS, AS LIQUIDS, AND IN SOLUTION. 1555

that the persistency of this band is not so marked as that of either the toluidines or the xylidines.

Benzylamine.-An $N / 1000$-solution transmitted the rays to about $\lambda 2290$ through a thickness of $30 \mathrm{~mm}$., but an $N / 100$-solution showed a moderately strong band, the curve of which has been drawn (Fig. 1). The position of this band is more towards the regions

Fig. 2.

Oscillation frequencies.

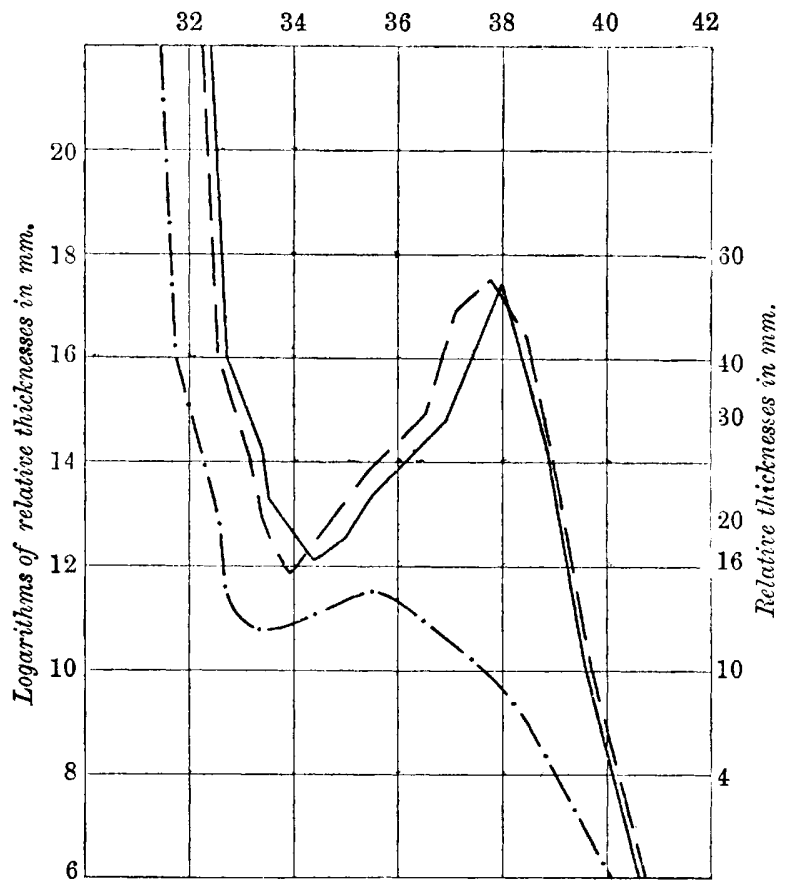

Continuous curve : N/1000-alcoholic solution of 0-3-xylidine. Dash curve : N/1000-alcoholic solution of m-2-xylidine. Dot and dash curve : N/1000-alcoholic solution of mesidine.

of greater refrangibility than in any of the substances herein described. It is of some importance to notice that the band corresponds in position and general form with the band $1 / \lambda 3850$ of diethylaniline.

\section{General Results of the Absorption Spectra of the Solutions.}

The general results of the investigation of the absorption spectra of the solutions are that (1) in the replacement of the hydrogen 
of the amino-group of aniline by alkyl groups, the persistence of the curve is considerably reduced; (2) when the hydrogen of the nucleus is replaced by alkyl groups the band becomes large and persistent; (3) in mesidine the band is not very strong or persistent; and (4) in benzylamine the band is fairly strong.

\section{Liquids.}

Hartley and Huntington (Phil. Trans., 1879, 170, I, 274) pointed out that it was only on dilution that the absorption bands of aromatic substances became visible. The thickness of the layer of liquid used by them was 0.75 inch.

In investigating the absorbent effect of liquids a special form of cell was used. It consisted of two quartz plates firmly cemented to a brass box or telescope. One of the quartz plates was attached to a movable micrometer screw, which could be moved by thousandths of a $\mathrm{mm}$., whilst the other plate was rigidly attached to a brass stage. A drop of the liquid to be investigated was placed between the two quartz plates, and these were then screwed together to a distance of $0.05 \mathrm{~mm}$. apart. The screw was turned so that the quartz plate attached to it was moved closer and closer to the rigidly placed plate until the distance between them was about $0.001 \mathrm{~mm}$. The light of the Cd spark was passed through the film of liquid for five minutes during each observation. Even through the thinnest films of about $0.001 \mathrm{~mm}$. no absorption bands were seen; and the following numbers state the regions where general absorption began under the above conditions, and through various thicknesses of the films.

\begin{tabular}{|c|c|c|c|c|c|c|c|}
\hline$A n$ & & Methyl & iline. & Dimeth & niline. & Ethyl & line. \\
\hline $\begin{array}{l}\text { Thickne } \\
\text { of film, } \\
\mathrm{mm} .\end{array}$ & $\lambda$. & $\begin{array}{c}\text { Thickness } \\
\text { of film, } \\
\mathrm{mm} .\end{array}$ & $\lambda$. & $\begin{array}{c}\text { Thicknes } \\
\text { of film, } \\
\text { mm. }\end{array}$ & $\lambda$. & $\begin{array}{c}\text { Thicknes } \\
\text { of film, } \\
\text { mm. }\end{array}$ & $\lambda$. \\
\hline 0.050 & 3185 & 0.050 & 3305 & 0.050 & 3310 & 0.050 & 3260 \\
\hline 0.030 & 3175 & 0.030 & 3295 & 0.030 & 3300 & 0.030 & 3250 \\
\hline 0.010 & 3160 & 0.010 & 3270 & 0.010 & 3280 & 0.010 & 3220 \\
\hline 0.004 & 3150 & 0.004 & 3260 & 0.004 & 3260 & 0.004 & 3210 \\
\hline 0.001 & 3140 & 0.001 & 3250 & 0.001 & 3270 & 0.001 & 3200 \\
\hline Diethy & iline. & o-Tolt & dine. & $\mathrm{m}-\mathrm{To}$ & dine. & $0-3-X$ & dine. \\
\hline 0.050 & 3325 & 0.050 & 3150 & 0.050 & 3180 & 0.050 & 3175 \\
\hline 0.030 & 3310 & 0.0 & & & & & \\
\hline 0.010 & 3285 & 0.010 & 31 & 0.0 & 31 & 0.0 & 3150 \\
\hline 0.00 & 3275 & 0.004 & 31 & 0.004 & 3130 & & 3140 \\
\hline 0001 & 3265 & 0.001 & 3095 & 0001 & 3125 & 0.001 & 3130 \\
\hline
\end{tabular}


HOMOLOGUES AS VAPOURS, AS LIQUIDS, AND IN SOLUTION. 1557

\begin{tabular}{cr}
\multicolumn{2}{c}{$\mathrm{m}-2-$ Xylidine. } \\
Thickness of \\
film, mm. & $\lambda$. \\
0.050 & 3200 \\
0.030 & 3180 \\
0.010 & 3160 \\
0.004 & 3145 \\
0.001 & 3140
\end{tabular}

\begin{tabular}{cc}
\multicolumn{2}{c}{ Mesidine. } \\
Thickness of \\
tilm, mm. & $\lambda$. \\
0.050 & 3250 \\
0.030 & 3210 \\
0.010 & 3160 \\
0.004 & 3140 \\
0.001 & 3130
\end{tabular}

Benzylamine.

$\begin{array}{cc}\text { Thickness of } & \\ \text { film, mm. } & \lambda . \\ 0.050 & 2745 \\ 0.030 & 2735 \\ 0.010 & 2730 \\ 0.004 & 2720 \\ 0.001 & 2710\end{array}$

General Results of the Absorption Spectra of the Liquids.

The more important points of interest in the above table are that (1) the constitution of the molecules of the various compounds influences the general absorption. For example, the positions of general absorption of the ortho-compounds are different from those of the isomeric meta-compounds; the differences in this respect are comparable with the differences observed in the absorption of their solutions; (2) isomeric substances like benzylamine, the toluidines, and methylaniline show differences in their positions of general absorption, which are also analogous to differences in the absorption of their solutions; (3) none of the substances when in the liquid condition exhibit absorption bands through the thinnest films of $0.001 \mathrm{~mm}$. thick.

It is not possible to imply very much from these numbers, as it is conceivable that films thinner than $0.001 \mathrm{~mm}$. are obtainable under greater pressure, which would allow more of the ultra-violet vibrations to pass through. On the other hand, such excessively thin films produced by pressure could no longer be considered as liquids. The physical condition of the molecules would then be more of the nature of a vapour under high pressure, and the results would hardly be comparable.

\section{Discussion of Results.}

In discussing the preceding observations we are confronted with the fact that the vapours of toluene, the xylenes, and mesitylene all exhibit absorption bands as described by Pauer, Grebe (loc. cit.), and by Hartley (Phil. Trans., 1908, A, 208, 475), and that the number of bands decreases as the number of methyl groups increases; but in the toluidines, not only do the bands of toluene vapour disappear, but also those characteristic of aniline vapour, and similar phenomena are observed in the xylidines and in mesidine. Further, the replacement of a hydrogen atom of the amino-group in aniline by an alkyl group produces a complete elimination of the bands characteristic of aniline vapour.

In the alcoholic solutions the type of absorption band is very similar in aniline and in the methyl- and ethyl-anilines, whereas

VOL. XCVII. 


\section{ABSORPTION SPECTRA OF ANILINE AND ITS HOMOLOGUES.}

in the toluidines and the xylidines the bands are stronger, different in position, and much more persistent than in either toluene, the xylenes, or aniline. The position and intensity of the band in benzylamine is also quite different from that of either toluene or aniline, but it corresponds in form and position with the more refrangible band of diethylaniline.

In the liquids, the general absorption is so great that the ultraviolet light is absorbed over the regions covered by the bands found in the solutions even at thicknesses of the liquids represented by $0.001 \mathrm{~mm}$.

The author has already discussed (loc. cit.) the absorption spectra of the vapours of pyridine and some of its derivatives from a consideration of the impacts of symmetrically and unsymmetrically oriented molecules. It seems to be possible to apply a similar explanation to the phenomena described in this communication. In the methyl- and ethyl-anilines the symmetrical orientation of the aniline molecule is distorted, and the vibrations of the more symmetrical aniline, which produce selective absorption, are destroyed. The vibrations producing the absorption bands in the vapours of toluene or the xylenes are wholly different from those producing the bands in aniline vapour; and in the vapours of the toluidines and the xylidines there is no trace of any of the series of bands. The symmetrical orientation of the atoms is absent in these compounds, and the vibrations no longer exercise a selective absorption of the radiant energy. The energy may be chiefly absorbed by the translatory energy of the molecules producing general absorption, which becomes more pronounced as the temperature and pressure are increased.

In the solutions, the solvent acts partly as a constraint on the vibrations of the molecules of the dissolved substance, partly as a barrier to the number of encounters, and partly as an absorbent of a portion of the radiant energy. The result of these influences is that the vibrations of the molecules are quite different from those of the vapours. The atoms of the molecules under these conditions vibrate or oscillate in a definite way characteristic of each substance; and molecules of the same type, for example, aniline and the methyl- and ethyl-anilines, exhibit bands having similar forms. They differ only in their relative positions and in their increased or decreased persistencies, which are regulated by the number, weight, and type of the substituted groups.

In the liquids, the molecules are more closely packed, and they exert a greater tensional effect on each other, so that the restraining influences on the vibrations are still more powerful. The radiant energy is absorbed by the molecules, for even when the film of 
liquid was only $0.001 \mathrm{~mm}$. thick, no bands of selective absorption were observed in any of the liquids. Nevertheless, the differences observed in the positions of general absorption of the ortho- and meta-compounds and in the isomeric substances, benzylamine, methylaniline, and the toluidines, indicate that, besides the weight of the molecule, the orientation is also a factor in determining the extent of the general absorption as distinct from selective absorption.

I have again to convey my thanks to the Government Grant Committee of the Royal Society, by whose assistance the larger portion of the cost of the apparatus used in these experiments was defrayed.

University Chemical laboratory, CAmbringe. 